\title{
Crack Detection via Differential Excitation and Gradient Orientation Based on Descriptor
}

\author{
Asna Baby $\mathbf{N}^{1}$, Harish Binu K $\mathbf{P}^{2}$ \\ ${ }^{1}$ MEA Engineering College, State Highway 39, Nellikunnu-Vengoor, Perinthalmanna, Malappuram, Kerala \\ ${ }^{2}$ Assistant Professor, MEA Engineering College, State Highway 39, Nellikunnu-Vengoor, Perinthalmanna, Malappuram, Kerala
}

\begin{abstract}
Crack detection and measurement in civil structures has been a constant field of research. In this paper, differential excitation, gradient orientation which is the important components of descriptor is proposed which is a new approach in the field of crack detection.Differential excitation component is defined as a function of two components. i.e., the relative intensity differences of current pixel with its neighbors and the intensity of current pixel whereas the orientation component is the gradient orientation of current pixel. It is done by introducing local spatial information, where an image is divided into number of blocks and histogram were computed for each blocks and concatenated. Based on this crack detection and further measurement of crack is done. Experimental result shows that this technique exhibits better performance and crack detection is done more efficiently.
\end{abstract}

Keywords: Descriptor, Differential Excitation, Gradient Orientation

\section{Introduction}

Crack detection and measurement plays as an inevitable role in the evaluation of fitness of structures like buildings, roads, pavements, etc. The presence of crack not only affects the visual appearance, but instead it transforms the matter of safety to be a big challenge. Based on the detected and measured crack further maintenance measures can be undertaken.

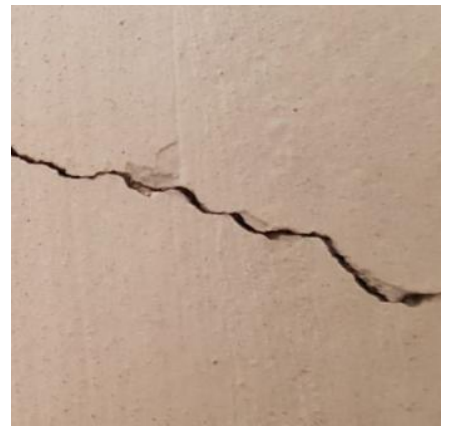

Figure 1.1: Crack on a surface wall

The main causes of crack formation are thermal movement, where it depends on number of factors like temperature variations, dimensions, and propertiesof materials used and the exposure of walls to direct solar radiation. Corrosion of reinforcement poor construction practices, poor structural design and specification also results in the occurrence of cracks. In addition to these, pavement distress is caused due to heavy vehicle traffic and changing weather conditions. So it becomes to be an essential task to monitor these defects before repair costs becomes too high.

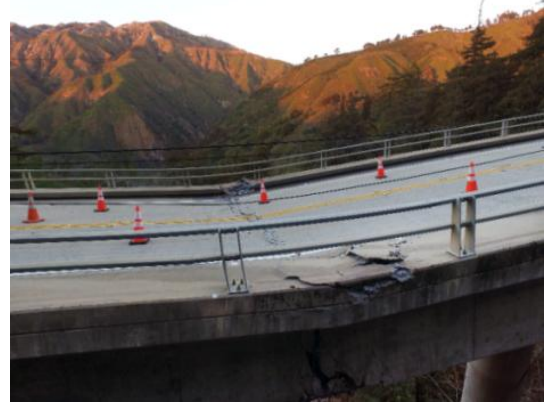

Figure 1.2: Effect of poor maintenance

The presence of crack gradually leads to shorter life span of structures. Many structures had collapsed due to this. For example, Can Tho Bridge in Vietnam (2007), Kadalundi River Rail bridge in India (2001), Rana Plaza Building in Bangladesh (2013). These tragical incidents are due to structural failures and highlight the need for periodical maintenance. The structural maintenance work includes repairing by concrete reinforcement, etc.

Crack detection and measurement can be done in many ways and generally as: human inspection where it make use of traditional measurement system on manually detected crack; microscopic inspection done by different special tools and machine vision inspection where automatic crack detection and measurement is done[1].

Here, in this paper, a texture descriptor based technique called differential excitation and gradient orientation is proposed for the purpose of crack detection and measurement process. This technique before is used in weber's Local Descriptor in fields like gender recognition from facial images[2].WLD is a texture descriptor that exhibits better performance when compared with other similar descriptors like LBP Gabor and SIFT[3].It is mainly done based on intensity difference of a pixel with the neighbouring pixel, is calculated and the corresponding histogram is built and hence the descriptor.Following this procedure the crack detection can be carried out where the 


\section{International Journal of Science and Research (IJSR) \\ ISSN (Online): 2319-7064}

Index Copernicus Value (2015): 78.96 | Impact Factor (2015): 6.391

presence of crack result in the intensity variation among the pixel orientation and the measurement process can be done.

This paper begins by describing the related works in Section II. Section III discusses the proposed architecture, the problem formulation, and the proposed algorithms. The experimental results and analysis are provided in Section IV. Finally, Section V presents the conclusion.

\section{Related Work}

In the past few years, many techniques to detect and measure cracks automatically have been studied. These techniques aims to assess the civil structures by monitoring cracks in a time period that provides safety for the users and it reduce the maintenance cost. Among these proposed techniques, approaches based on image analysis, neural networks, pattern recognitionand particle filtering have emerged as the most efficient ones. For example, LBP (local binary pattern), a technique used for texture classification based on the grey-scale and rotation invariant operator from images has been used for crack detection. This method uses a LBP operator thatdescribes the neighbouring pixel[4] classifying local neighbours into smooth and rough areas of an image and then the areas with coarse texture are further explored.

Moreover, neural networks, i.e., back propagation neural networks which performs gradient descendent within solution vector space towards global minimum of error is used for the purpose of crack detection and further it classifies the detected pavement crack as either linear or alligator ones [6].

Wavelet transform [5] which is most popular approach for time-frequency transformations is another approach for crack detection process. It performs local analysis of signals and reveals hidden aspects of data and is used for pavement distress image compression noise reduction [9].Scale and translation are the two basis parameters of wavelet analysis [2]

STRUM (Spatially Tuned Robust Multi feature) classifier. i.e., an automated crack detection algorithm is also used which demonstrates the result on robotic bridge scanning system where it uses robust curve fitting that spatially localizespotential crack regions even in the presence of noise.

Study through different edge detection techniques like canny filter, sobel method shows that each method exhibits different performance for each surfaces done by updating the assigned weight to each particles in the sample set.Particle filtering [1] is another approach for automating crack detection process where it approximates the solution. Particle filter is a non - parametric state estimator.i.e., it does not make any assumption on measurements. The particle filter is evolved from Monte - Carlo method. The main aim is to find the distribution of $f$ of the state vector and starting from an initial distribution $g$, estimates that closely approximates the real value of state vector $x$ [2].
In this section III. A, gives an overview of the architecture of proposed crack detection process and section III. B explains the basic procedure of differential excitation, gradient orientation, histogram and descriptor based on which the crack is detected.

\section{A. Overview}

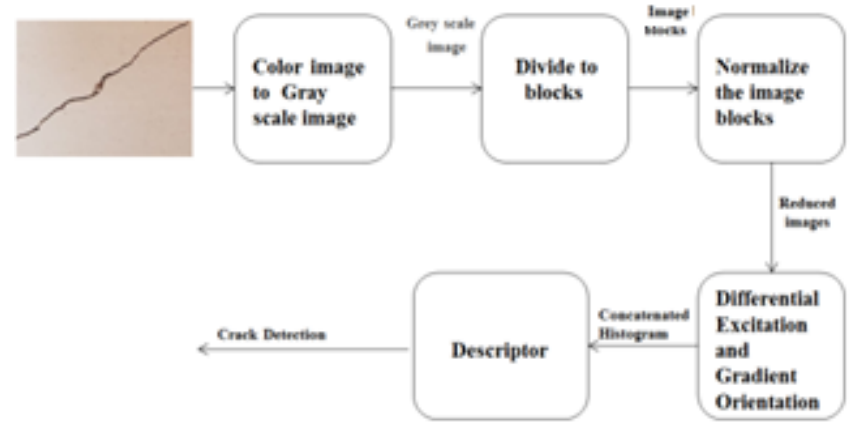

Figure 3.1: Overall structure of crack detection system B. Crack Detection

Differential excitation and gradient orientation are the two approaches, are introduced for the purpose of crack detection.et.al[3] defines the descriptor for the purpose of feature extraction where the descriptor represents an image as histogram of gradient orientation and differential excitation.It exhibits several properties like robustness to noise and illumination changes and elegant detection of edges and moreover powerful image representation.

\subsection{Differential Excitation}

Differential excitation component for a given pixel is computed based on the ratio between the two terms.i.e., the relative intensity differences of a current pixel with its neighboring pixel and the intensity of the current pixel.With the differential excitation component, the local salient patterns in the input image and the intensity differences between its neighbors and a current pixel are considered as the changes of current pixel [8].

Differential excitation used in crack detection is based on the intensity difference of pixel.i.e., Calculation of differential excitation $\varepsilon\left(x_{p}\right)$ of pixel $x_{p}$ involves finding intensity difference with the neighboring pixel $x_{i}$ where $i=$ $1,2 \ldots$. It can be calculated as:

$$
\Delta I_{i}=I_{i}-I_{p}
$$

In the next step,ratio of total intensity difference of $x_{p}$ with its neighboring pixel $x_{i}$ is determined as:

$$
f_{\text {ratio }}=\sum_{i=0}^{p-1} \frac{\Delta I_{i}}{I_{p}}
$$

An arctangent function is used inorder to compute $\mathcal{E}\left(x_{p}\right)$ and this function isused since it can limit the output to prevent it from quick increase or decrease when the input becomes larger or smaller.

Further, an arctangent function is applied on the above Equation which enhances the robustness against the noise. It is given as:

$$
f_{\text {arctan }\left[f_{\text {ratio }} x_{p}\right]}=\arctan \left[f_{\text {ratio }}\left(x_{p}\right)\right]
$$

\section{Proposed System}




\section{International Journal of Science and Research (IJSR)}

ISSN (Online): 2319-7064

Index Copernicus Value (2015): 78.96 | Impact Factor (2015): 6.391

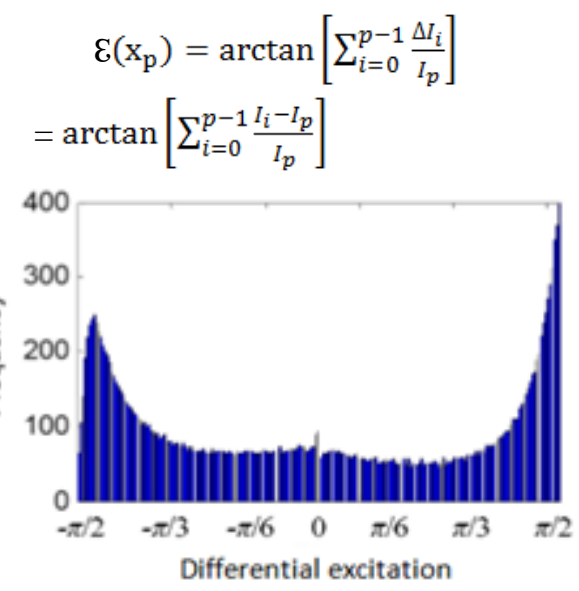

Figure 3.2: The average histogram on 2000 texture images

[3]

The Figure 3.2.shows an average histogram plotted for differential excitation on 2,000 texture images. It is clear that there are more frequencies at the two sides of average histogram. i.e., $\left[\frac{-\pi}{2}, \frac{-\pi}{3}\right]$ and $\left[\frac{\pi}{3}, \frac{\pi}{2}\right]$.

It is because of two factors: delimitation effect of the arctangent function and the computing approach of differential excitation $\mathcal{E}\left(x_{p}\right)$ of a pixel. i.e., sum of difference ratios of $p$ neighbors against a central pixel. The differential excitation $\mathcal{E}\left(x_{p}\right)$ may take a minus value if the neighbour pixel intensities are smaller than the current pixel intensity.Hence we attempts to preserve more discriminating information in comparison to using the absolute value of $\mathcal{E}$ $\left(x_{p}\right)$.

The differential excitation $\mathcal{E}\left(x_{p}\right)$ will be either positive or negative where each value indicates that the current pixel is darker than its surrounding pixel and is lighter than the surrounding respectively.

\subsection{Gradient Orientation}

The next step is the gradient orientation.Gradient orientation for a pixel can be calculated as:

$$
\theta\left(x_{p}\right)=\arctan \left[\frac{I_{73}}{I_{51}}\right]
$$

where $I_{73}=I_{7}-I_{3}$ is the intensity difference of two pixels on the left and right of the current pixel $x_{p}$, and $I_{51}=$ $I_{5}-I_{1}$ is the intensity difference of two pixels directly below and above the current pixel, $\theta \in\left[-\frac{\pi}{2}, \frac{\pi}{2}\right]$

The gradient orientations are quantized into $\mathrm{T}$ dominant orientations. The quantization function is given as :

$\phi_{t}=\frac{2 t}{T} \pi$

Where, $\mathrm{t}=\bmod \left(\left\lfloor\frac{\theta^{\prime}}{2 \pi / T}+\frac{1}{2}\right\rfloor, T\right)$

And $\theta^{\prime} \in[0,2 \pi]$ and is defined in terms of gradient orientation computed.
The key idea of representing an image by using the histogram of the corresponding differential excitation and gradient orientation is widely useful in object detection, biologically plausible vision systems and in image recognition. After computing each pixels differential excitation and then computing the pixels gradient orientation, then find the histogram of the corresponding result.

The main idea of finding the histogram of oriented gradient descriptor is that the shape within the image local object appearance and can be described by the distribution of intensity gradients and its edge directions. Corresponding to each dominant orientation $\phi_{t}: t=0,1,2 \ldots T$ 1differentialexcitations are organized as a histogram $H_{t}$. Then each histogram $H_{t}: t=0,1,2 \ldots T-1$ is evenly divided into $M$ sub histograms $H_{m, t}: m=0,1,2 \ldots M-1$. These histograms form a histogram matrix, where the column represents the dominant orientation $\phi_{t}$. Each row of this matrix is concatenated as a histogram $H_{m}=\left\{H_{m, t}: \mathrm{t}=0,1\right.$, $2 \ldots T-1\}$. Subsequently, histograms $H_{m}: m=0,1,2, \ldots, M-1$ are concatenated into a histogram $H=\left\{H_{m}: m=0,1,2, \ldots\right.$, $M-1\}$. This histogram is gives the descriptor and it involves free parameters: $\mathrm{T}$, the number of dominant orientations, $\mathrm{M}$ the number of segments of each histogram corresponding to a dominant orientation.

\subsection{Descriptor}

The basic descriptor represents an image as the histogram of differential excitation which is organized according to the dominant gradient orientations. Here, in the histogram differential excitations are collected which is based on their corresponding values and the gradient orientations. The spatial location is a very important factor for getting much better description else the descriptor cannot discriminate the regions efficiently. Hence, discriminatory power is enhanced by introducing the spatial information to the descriptor. By using this descriptor, the feature is extracted. Correlation is a simple classifier used here which is used for the purpose of comparison. The images are divided into a number of blocks followed by computing the histogram for each block and is concatenated them in order to form the descriptor. Here, the size of the blocks is a very important factor. As the size of blocks decreases the result will be more accurate.

\section{Experimental Analysis}

The proposed algorithm for crack detection is implemented by using the Matlab2014 a(64 bit) with operating system 10 . As a first step, the input image is selected for the purpose of implementing the proposed approach.

\subsection{Histogram}




\section{International Journal of Science and Research (IJSR)}

ISSN (Online): 2319-7064

Index Copernicus Value (2015): 78.96 | Impact Factor (2015): 6.391

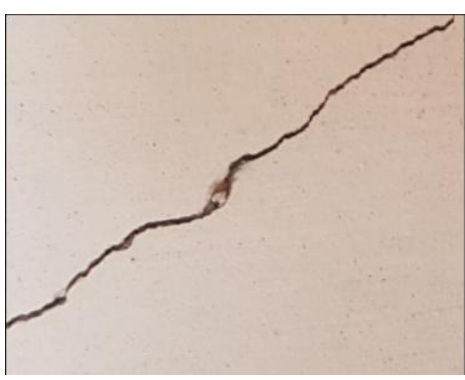

Figure 4.1: The input image

The above figure 4.1 shows the input image, which is in the RGB form and further it is converted into grey scale image. On this image different excitation and the gradient orientation is done for deriving the descriptor.

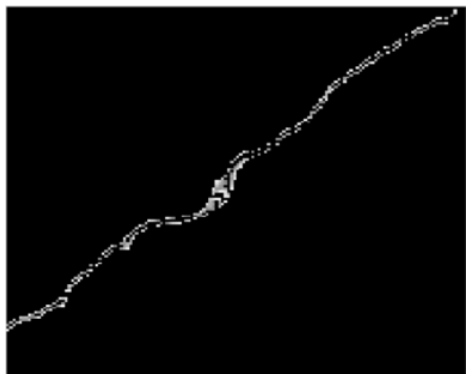

Figure 4.2: Result

Figure 4.2 shows the desired output of the input after undergoing the process of descriptor with the differential excitation and gradient orientation.

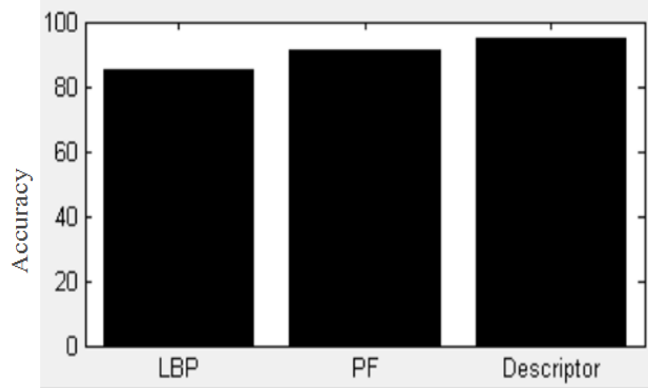

Figure 4.3: Performance Comparison

The above figure 4.3 shows the performance comparison graph among the techniques like LBP (Local Binary Pattern), PF(Particle Filter) and the proposed approach using the input database. It shows that the proposed approach outperforms the LBP and PF on the basis parameters like accuracy, and execution time.

\section{Conclusion}

This paper aims at introducing a new approach in the field of crack detection there by increasing the accuracy with low computational time. The technique used here, mainly comprises the combination of differential excitation, gradient orientation with their corresponding histogram followed by the descriptor with local information which enhances the discriminatory power. It is done by dividing the image into a number of blocks. It exhibits better performance in terms of accuracy when compared with the existing crack detection methods like LBP (Local Binary Pattern) and particle filter.

\section{References}

[1] Romulo GonçalvesLins and Sidney N. Givigi, “ AutomaticCrack Detection and Measurement Based on Image Analysis”, IEEE Transactions On Instrumentation And Measurement ,0018-9197,2016.

[2] IhsanUllah, Muhammad Hussain,Ghulam Muhammad, HatimAboalsamh and George Bebis,"Gender Recognition From Face Images With Local WLD Descriptor” ,IWSSIP ,11-13 April 2012.

[3] JIE Chen,Shiguang Shan, Chu He,Guoying Zhao,MattiPietikainen,Xilin Chen, Wen Gao,"WLD: A Robust Local Image Descriptor",IEEE Transactions on Pattern Analysis and Machine Intelligence, TPAMI2008-09-0620,2009.

[4] Y. Hu and C.-X. Zhao, "A local binary pattern based methods for pavement crack detection," J. Pattern Recognit. Res., vol. 5, no. 1, pp. 140-147, 2010.

[5] P. Subirats, J. Dumoulin, V. Legeay, and D. Barba, "Automation of pavement surface crack detection using the continuous wavelet transform," in Proc. IEEE Int. Conf. Image Process., Oct. 2006, pp. 3037-3040 .

[6] Li, Sun, Ning, Tan,"Automatic Pavement Crack Recognition Based on BP Neural Network", Promet Traffic\&Transportation, Vol. 26, 2014, No. 1, 11-22 .

[7] G. G. Rigatos, "Particle filtering for state estimation in nonlinear industrial systems," IEEE Trans. Instrum. Meas., vol. 58, no. 11, pp. 3885-3900, Nov. 2009

[8] D.G. Agrawal, Pranoti M. Jangale, "Dynamic Texture Feature Extraction Using Weber Local Descriptor," Journal of Engineering Research and Applications, Vol. 4, March 2014, pp.502-506

[9] Asna Baby N, Harish Binu K. P., "Survey on Automatic Crack Detection and Measurement Techniques", International Journal of Science and Research (IJSR),ISSN 23197064, Volume 6 Issue 4, April 2017

[10]Henrique Oliveira and Paulo LobatoCorreia, "Supervised Crack Detection and Classification in Images of Road Pavement Flexible Surfaces" ,Recent Advances inSignalProcessing

[11] Mohammed Ali Lotfollahi-Yaghin and Mohammed AminHesari, "Using Wavelet in Crack Detection At the Arch Concrete Dam under Frequency Analysis with FEM ",WorldApplied Sciences Journal 3(4). ,2008,pp.691-704

\section{Author Profile}

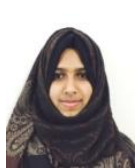

Asna Baby N received her B.Tech degree in computer science and engineering from the KMCT College of Engineering For women, Kerala, in 2015. Right now she is pursuing her M.Tech degree in computer science at MEA Engineering College, Kerala from 2015 to 2017. Her research interests lie in Image Processing.

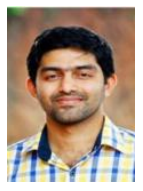

Harish Binu K.P., Assistant Professor, MEA Engineering College, Perinthalmanna. Received the B.Tech degree in Computer Science\& Engineering from NSS Engineering College, Palakkad, University of Calicut and M.tech degree in Computer Science from College of Engineering, Cochin 